\title{
Ilse Maria Lenz \\ Der große Unterschied und die kleine Gleichheit: Zur Bedeutung ler geschlechtlichen Arbeitsteilung für die japanischen industriellen Beziehungen
}

Um die spektakuläre soziale und wirtschaftlicheEntwicklung Japans zu begreifen, ist eine Beschäftigung mit der geschlechtlichen Arbeitsteilung und der Stellung der Frau unerläßlich. Allerdings stößt der Blick »von außen« zunächst auf von Männern geprägte Strukturen: die häufig untersuchten (männlichen) Facharbeiter, die mit ihrem Arbeitsethos den japanischen Wirtschaftserfolg mitbegründet haben sollen, die »paternalistischen« (männlichen) Manager, die (überwiegend männlichen) Studenten an den Elitehochschulen, die die »Examenshölle der Schule« überstanden, um nach dem Universitätsexamen in die Großbetriebe einzutreten usw. Doch bauen diese in Europa vielfach beleuchteten Zusammenhänge auf der geschlechtlichen Arbeitsteilung in der Familie und im Bereich der Lohnarbeit auf. Erst die Tatsache, daß japanische Hausfrauen die »Familienarbeit « — vom Einkaufen und Kochen bis zur alltäglichen Versorgung der schulgestreßten Kinder - fast alleine erledigen, ermöglicht die Hinwendung zu und vorrangige Loyalität der männlichen »Stammarbeiter « gegenüber ihren Betrieben. Die täglichen Überstunden, die Arbeitseinsätze in entfernten Städten, die Angehörigen des Managements und der Kernbelegschaft nicht selten abverlangt werden - all dies setzt voraus, daß die »Familienväter《 in der Familie weitgehend abkömmlich sind.

Diese häusliche geschlechtliche Arbeitsteilung ist eine der Voraussetzungen der betonten geschlechtlichen Spaltung des Arbeitsmarktes, die zugleich durch betriebliche Regelungen befestigt und entsprechend den betrieblichen Anforderungen laufend angepaßt und teils verfeinert wird (vgl. Lenz 1987). Die Geschlechtsgrenze ist die wichtigste Fragmentierungslinie zwischen den Belegschaftsgruppen, obwohl auch ethnische und soziale Diskriminierung z.B. gegenüber in Japan ansässigen Koreanern oder der unterdrückten Minderheit der burakumin eine gewisse Rolle spielen (Ernst 1980). Aber angesichts der weitgehend homogenen Voraussetzungen der Mehrheit der Beschäftigten, die die gleichen mittelschichtorientierten Normen der Massenkultur teilen und mindestens über einen Mittelschul- oder Oberschulabschluß verfügen, wurde in der industriellen Expansionsphase nach 1955 die »Geschlechtsgrenze « auf dem Arbeitsmarkt und in den industriellen Beziehungen als Kriterium der hierarchischen Abstufung betont und systematisch ausgebaut. Häufig werden in der deutschen Öffentlichkeit die (überwiegend männlichen) Kernbelegschaften der Großbetriebe mit »dem japanischen Arbeiter« schlechthin gleichgesetzt; zu Beginn der 1980er umfaßten sie jedoch knapp unter $30 \%$ aller abhängig Beschäftigten (Kemmochi 1983: 225). Hinter und unter ihnen stehen die wenig beachteten instabil Beschäftigten, d.h. Männer und Frauen in Klein- und Mittelbetrieben, Arbeiterinnen in der Großindustrie, die bis zur Ehe oder bis zum ersten Kind erwerbstätig sind, sowie die wachsende Zahl von Teilzeitarbeiterinnen (Bergmann 1983; Lenz 1987). 
Die japanische Entwicklung verweist also auf die Notwendigkeit einer integrierten Perspektive von gesellschaftlicher Arbeitsteilung, die die betrieblichen Grenzen überschreitet. Zugleich aber verdeutlicht sie historisch-kulturelle Variationen zwischen den kapitalistischen Industriegesellschaften, die durch patriarchatskritische Untersuchungen erfaßbar werden: der »japanische patriarchalische Kapitalismus« unterscheidet sich in wesentlichen Zügen von seinem westlichen Gegenstück. Im folgenden will ich diesen Unterschieden, aber auch den Gemeinsamkeiten vor allem anhand der Frage nachgehen, wie der Wert der Polarisierung des Geschlechtsunterschiedes in Japan in der betrieblichen und überbetrieblichen Arbeitspolitik umgesetzt wurde. Was bedeutet die Zuschreibung von unterschiedlichen, gegeneinander kaum durchlässigen Bereichen für beide Geschlechter, nach der der moderne Haushalt auf die Mutter, der Betrieb auf den männlichen Lohnarbeiter zentriert sein soll, während das Gleichheitspostulat i.a. erst auf Grundlage dieser Zuschreibungen erhoben und ihm nur eine begrenzte Geltung gegeben wird? Die »Gleichheit des Sexus« in der Industriegesellschaft, die z.B. Illich so heftig beklagt, hat sich in Japan nicht eingestellt (vgl. Illich 1984). Eine Auseinandersetzung mit Formen patriarchalisch-kapitalistischer Verhältnisse, die einer anderen Entwicklungsgeschichte und Kultur entspringen, kann also auch Anlaß dazu geben, über den Wert und die Grenzen »unseres « auf der Gleichheits. Tradition begründeten Emanzipationsverständnisses nachzudenken ${ }^{1}$. Allerdings setzt dies voraus, nicht von vornherein die europäische Elle der Gleichheit anzulegen, um dann auf die »unterdrückten Japanerinnen« herabzusehen, sondern sich auf die Realität der Japaner/innen in ihrer Spannung zwischen »Lebenswelt und System « erst einmal einzulassen. Dabei stößt die sozialwissenschaftliche Japan-Diskussion auf Probleme, die ich unter Bezug auf Edward Saids brillante Untersuchung des »Orientalismus« in der europäischen Asienwissenschaft skizzieren möchte: Unter »Orientalismus« versteht Said die Art und Weise, mit der Europa herkömmlich den Orient betrachtet hat. Die Strukur dieses Diskurses war durch eine selektive Wahrnehmung der asiatischen Gesellschaften bestimmt, die durch europäische Sozialverhältnisse, Konflikte und Wunschprojektionen gespeist wurde: sprechende Beispiele dafür sind die in Europa entworfenen Bilder der asiatischen Frauen von der sanften »Madame Butterfly« bis zur thailändischen »mandeläugigen Sexgespielin «, die vor dem Hintergrund der europäischen Frauenbewegungen projiziert wurden. Saids Ideologiekritik zeigt in einer gründlichen Durchsicht der europäischen Asienliteratur drei Merkmale des 》Orientalismus« auf (Said 1980):

1. Es besteht ein Machtverhältnis gegenüber den seit dem 18. Jh. auch real unterlegenen asiatischen Gesellschaften, das șich darin ausdrückt, daß die europäischen Mächte nun einen »Blick von oben « auf den Orient annehmen.

2. Der Orient wird als eng verbunden mit Europa und doch als das völlig Fremde, »das Andere«, dargestelit. Mit einer positiven Wertung geschieht dies in den Diskursen über die »Weisheit des Ostens « — oder wie schon erwähnt die »ganz anderen« geheimnisvollen asiatischen Frauen. Aber auch das Stereotyp des economic animal im »A rbeitsstaat Japan«, das nur für die Firma lebe und keine persönlichen Emotionen habe, gerät zu einer negativ unterlegten Neuauflage dieses Verhältnisses von geforderter Nähe und »ganz Anderem«.

3. Der »Osten « kann sich nicht selbst darstellen, hat er doch die Fähigkeit, historisches, aussagebestimmendes Subjekt zu sein, für den »Westen« verloren. Vielmehr wird er repräsen- 
tiert in Form von Vor-bildern, vorgeformten Imaginationen und Urteilen, und wird immer wieder nach »westlichen Bedürfnissen « interpretiert.

Eben diese drei Kennzeichen des »westlichen« Asien-Diskurses — das Machtverhältnis, die Auffassung vom Unterlegenen als einem völlig Fremden und die Repräsentation als Objekt für das geschichtstragende Subjekt - finden sich auf einer anderen Ebene im gesellschaftlichen und wissenschaftlichen Diskurs über Frauen wieder. $\gg$ Der Blick von oben « be herrschte die Wahrnehmung der Frau, häufig ohne das zugrundeliegende Machtverhältnis zu reflektieren. Frauen galten als das »andere Geschlecht« (Beauvoir) im Rahmen männlich zentrierter Maßstäbe. Sehr lange wurden sie im gesellschaftlichen Diskurs nur repräsentiert, ohne daß sie selbst zu Wort kommen konnten oder ihre Stimmen gehört wurden. In der herkömmlichen Japanliteratur überblenden und vermischen sich vielfach wissenschaftlicher Androzentrismus (Zentrierung auf »männliche« Werte und Sichtweisen) und Orientalismus. So werden die Frauenaspekte im »ganz anderen Japan « entweder gar nicht wahrgenommen oder auf ein Ghetto des »Sonderproblems Frau《 eingeschränkt. Gravierender aber ist die Fremdrepräsentation der Japanerin, ohne daß ihre Perspektive aufgenommen würde. Die Weltsicht japanischer Frauen ist in Europa noch eine weithin unbe kannte Welt.

In dieser Fremdsicht dominiert entweder die These von der reinen »Unterdrückung« oder aber umgekehrt die Annahme der kulturspezifischen Akzeptanz, die die Japanerinnen für ihre Sonderrolle als Hausfrau und Mutter aufbringen sollen. In beiden entgegengesetzten Annahmen werden die Widersprüche und Brüche, die die Japanerinnen in ihrem Lebenszusammenhang erfahren und die sich in erheblichem Druck auf die in der Epoche des hohen Wachstums eingespielte Balance zwischen Heim und Betrieb ausdrücken, nicht mehr sichtbar. Bei der Suche nach den Frauen als Subjekt stellt sich das Problem, ihre widersprüchliche Lebenswirklichkeit »von innen « her zu begreifen. Dies geht nicht in einer Perspektive »von oben und außen «, die Frauen nur im Zusammenhang mit dem herrschenden Frauenbild und den entsprechenden Normen wahrnimt. Sie kann als Perspektive des »Empfangsraums « bezeichnet werden, in dem die lächelnde Japanerin dem Gast von auBen Tee serviert (und folglich nur als untergeordnete Dienstleistende gesehen wird). Die Wahrnehmung muß sich aber auch auf »weibliche Orte« richten, also eine Perspektive z.B. »aus der Küche«, »aus der Frauengruppe an der Montage« her usw. entwickeln.

\section{Die nachholende japanische Emtwicklung und Veränderungen in der Stellung der Fran}

Die japanische kapitalistische Entwicklung kann jenseits der aktuellen Fragestellungen, die der verstärkten Konkurrenz mit der »Wirtschaftsmacht Japan « entspringen, ein vergleichendes Interesse unter dem Aspekt der Fragen sozialer Evolution beanspruchen ${ }^{2}$. Dies gilt auch und gerade für die geschlechtliche Arbeitsteilung und Machtverhältnisse. Einerseits stoßen wir im Vergleich zur langzeitlichen Herausbildung der burgerlichen Gesellschaft in Europa auf eine Zusammendrängung und »Verquickung« von Entwicklungsstufen, wie wir sie kennen. Andererseits problematisiert das Verhalten der wichtigsten Akteure in der Modernisierung Japans herkömmliche Vorstellungen eines geradlinigen Fortschritts von 
der Tradition zur Moderne: Denn die Tradition wird selektiv aufgenommen, umgeformt und in moderne Institutionen und Organisationen überführt, wobei andere traditionelle Bereiche brach liegen oder verschüttet werden. Diese Kombination »traditioneller « Elemente bedeutet eine »Kultursynthese « in der Modernisierung, die jedoch einen Bruch mit der Geschichte beinhalten kann. Ein Beispiel stellt die Entwicklung der industriellen Bezichungen in der ersten modernen Großindustrie Japans, der Baumwollindustrie, dar: Unter Berufung auf die japanische patriarchalische Tradition wurden die Arbeitsverträge für die Arbeiterinnen mit ihren Vätern oder Haushaltsvorständen abgeschlossen, die auch einen größeren Lohnvorschußerhielten. Aber die herkömmlichen Baumwollspinnereien, die um 1870, vor der Einführung der »westlichen« großen Spinnmaschinerie, für den Inlandsmarkt produzierten, hatten offenere Arbeitsmärkte, in denen auch Arbeiterinnen als weitgehend eigenständige Vertragspartner auftraten. ${ }^{3}$.

Die japanische Modernisierung beruht also auf einer Seiektion, Synthese und Neuschaffung eigener »herkömmlicher《 und neuer »westlicher« Elemente, die für die nachholende wirtschaftliche und soziale Entwicklung Japans mobilisiert wurden. Die staatlichen und wirtschaftlichen Eliten woliten sowohl in der Konkurrenz mit den westlichen Mächten bestehen als auch durch eine autoritäre politische Kultur die Spannungen dieses Prozesses abfangen und kanalisieren. Die Verdichtung und Zusammendrängung und die Selektions- und Syntheseprozesse der Modernisierung bedeuteten, daß sich in Japan die Herausbildung europäischer bürgerlicher Gesellschaften mit ihren Idealen des Individualismus und der Freiheit nicht wiederholte (Ishida 1987). Hier können nur einige Aspekte dieser komplexen Entwicklung gestreift werden. Beck-Gernsheim beispielsweise gibt zu bedenken, ob in Europa die Herausbildung des autonomen bürgerlichen Individuums sich nicht auf die Männer beschränkte; die Frauen blieben zunächst in das Kollektiv der »modernen Familie« gebannt, ehe sie in den letzten Jahrzehnten ihre individuellen Ansprüche und Ziele lauter äußerten und auf dem Arbeitsmarkt stärker auftraten (Beck-Gernsheim 1986). In Japan betonten die politischen und wirtschaftlichen Eliten ausgewählte japanische Gemeinschafts-Elemente, während sie sie zugleich z.B. über rechtliche Festschreibung oder durch ideologische Neufassung veränderten und den Modernisierungszielen anpaßten: Deutlich tritt dies in der Staats- und Familienlehre zutage. Nach einigen liberalen Experimenten vor allem unter französischem Einfluß wurde im BGB von 1898 das »Haus« (ie) und nicht das Individuum als Grundeinheit der Gesellschaft gesetzt. Das Haus, d.h. die patriarchalische Mehrgenerationenfamilie, wurde im neuen Recht, in Anlehnung an das Hausrecht der Samurai, einem männlichen Haushaltsvorstand unterstellt; damit wurde das bäuerliche Volksrecht aufgehoben, nach dem auch Frauen diese Stellung innehatten (vgl. Lenz 1984: 122 ff.). Wichtig ist hier, daß diese Veränderungen die Individuation der Männer und Frauen hemmten; beide Geschlechter orientierten sich weiter an Formen der Gemeinschaft, vor allem an dem »Haus« und der Dorfgemeinde, auch wenn diese Bindungen bei den Frauen stärker ausgeprägt waren. Auch gegenwärtig setzen sich diese gemeinschaftiche Orientierung und ihre stabilisierende Funktion z.B. darin fort, daß die Großbetriebe von ihren mämnlichen Stammbelegschaften ein hohes »Bewußtsein für die Betriebsgemeinschaft« (kigyo kyodotai ishikl) erwarten.

Während die klassischen Prinzipien der bürgerlichen Revolution in Europa, also Freiheit und Gleichheit der Individuen, in der japanischen Modernisierung nur partiell aufgegriffen 
wurden, strömte aus der Tradition der bäuerlichen Schichten die Idee der Gleichheit von Angehörigen überschaubarer Kollektiven, z.B. der Dorfgemeinschaften, in die politische Kultur ein. Diese Strömung wurde nach 1945 spürbarer, als die »Nachkriegsreformen« (Bodenreform, Abschaffung des alten Familienrechts und des Haushaltsvorstandes, Gleichheitsgebot der Verfassung) überlebte Herrschaftspositionen beiseite gefegt hatten. Diese »dörfliche« Form der Gleichheit beinhaltet nicht, daß aufgrund eines abstrakten Gleichheitsanspruchs alle Mitglieder der Gesellschaft gleiche Rechte hätten; vielmehr besteht die Gleichheit darin, daß die Einzelnen als Mitglieder einer - begrenzten oder sogar abgegrenzten - Gemeinschaft anerkannt werden, wobei ihnen aus dieser Mitgliedschaft unterschiedliche Rechte zukommen können. Gleich sind sie ferner in dem geforderten Gemeinschaftsbewußtsein. Diese $\gg$ Gleichheit zur Gemeinschaft $«$, die sich nicht allgemein auf 》Gesellschaft《 erstreckt, findet sich m.E. in der Betriebsbindung der japanischen Stammbelegschaften und in der betrieblichen Stellung der Frau wieder. Doch konfligiert sie in sozialen Auseinandersetzungen, z.B. den zahlreichen Arbeitsprozessen von Frauen für Gleichheit arn Arbeitsplatz oder in der Praxis von gewerkschaftlichen Gruppen, häufig Minderheitsgewerkschaften, mit Konzepten geselischaftlicher Gleichheit. Die Entwicklung des Gleichheitsdenkens aus diesen unterschiedlichen Strömungen dürfte ein wichtiges Thema der nächsten Jahre darstellen 4 .

In der Zusammendrängung von Entwicklungsstufen, die die nachholende japanische Entwicklung erforderte, bildete das Fortwirken des Standesdenkens die Rückseite der Medaille der hier umrissenen Gemeinschafts-Orientierung. Während die alten feudalen Stände um 1868 weitgehend aufgehoben wurden, blieb der aus kollektiven Zugehörigkeiten erwachsende Stand oder Status (mibun) ${ }^{5}$ ein wichtiges Kriterium zur Einordnung der Einzelnen, deren Mobilität in der Modernisierung sich ansonsten ungeheuer erweitert hatte. Dies zeigt sich z.B. in der Bedeutung von Status-Elementen in der Spaltung der Arbeitsmärkte: Bis zur Gegenwart wirken sich der Status der Einstellung wie die Beschäftigung direkt nach dem Abschluß der Ausbildung, oder aber der Status einzelner Beschäftigten-Gruppen, wie etwa der Teilzeitarbeiter/innen, auf Beschäftigungssicherheit, Aufstieg und Entlohnung aus. Wie in Europa ist das zugrundeliegende Argument des Managementes ökonomischer Art: Nur eine Vollzeit- und Dauerbeschäftigung rechtfertige hohe Investitionen in die betriebliche Ausbildung. Aber die Schärfe der Abgrenzung der Gruppen geht m.E. auch auf das Wirken des 》Status« in diesem Sinne zurück; sie zeigt sich darin, daß Übergänge nach oben auch bei vorhandener Qualifikation kaum möglich sind. Das Vorrücken vom Status Teilzeitarbeit in den der Dauerbeschäftigten, dem im europäischen Kontrastfall der Wechsel von Teilzeit- zu Vollzeitarbeit entspräche, ist undenkbar. Ebenso werden in einer Reihe von Betrieben Unterschiede zwischen den Beschäftigten, die nach der Schulausbildung dort eintraten, und »Betriebswechslern« gemacht. Wie im folgenden Abschnitt gezeigt werden soll, ist das weibliche Geschlecht ein grundlegender Faktor bei dieser Statuszuschreibung.

In der Selektion und Synthese herkömmlicher und westlich-moderner Elemente bildeten sich auch aufeinander aufbauende Stufen des Patriarchats als ideologische und materielle Verhältnisse heraus: Das Haus (ie) im japanischen Kaiserreich des späten 19. und frühen 20. Jhs. stützte sowohl die Unterdrückung der Frau als auch die allgemeine Unterordnung 
der Untertanen in dem »Familienstaat« legitimatorisch ab. Zugleich beruhte der nachholende exportorientierte Entwicklungsweg Japans auf einer patriarchalischen Arbeitsteilung in Industric und Haushalt (vgl. Lenz 1984, Oba/Ujihara 1969: 345-360). Die patriarchalische Strukturierung beider Bereiche steht in wechselseitigem Zusammenhang und verstärkte sich gegenseitig. In den ersten Industrialisierungsphasen mußten die Leichtindustrien, vor allem die Textilindustie, die notwendigen Devisen für den Aufbau von Schwerindustrie und Rüstungsindustrie, mitsamt dem dafür benötigten Technologietransfer, den Maschinen und Rohstoffen einbringen. In Exportindustrien arbeiteten überwiegend junge Frauen.

Dem hohen Frauenanteil an der Industriearbeiterschaft der Frühphasen, der von ca. $60 \%$ 1910 auf ca. $50 \% 1930$ (vgl. Lenz 1984: 199 ff.) zurückging, liegen die herkömmlich hohe Erwerbsquote der Frauen in Landwirtschaft und Kleinhandel, die Frauenbeschäftigung in einigen binnenmarktorientierten Kleinindustrien für den Alltagskonsum und die exportorientierte Industrialisierungsstrategie zugrunde. Gerade die modernen Exportindustrien wiesen damals eine betonte geschlechtliche Arbeitsmarktspaltung auf: Mädchen und junge Frauen, die teils von ländlichen Haushalten migrierten, wurden zu Niedriglöhnen und im internationalen Vergleich sehr langen Arbeitsstunden beschäftigt. Während sich Z.B. in der Metallindustrie allmählich erste Modelle der Dauerbeschäftigung für männliche Arbeiter herausbildeten, blieben in den Exportindustrien, die zumeist weibliche Arbeitskräfte bis ca. fünf Jahren oder häufig bis zur Ehe beschäftigten, Kurzzeitverhältnisse bestimmend. Für Arbeiterinnen waren also weder berufsqualifizierende Ausbildung und weiterführende Aufstiegschancen, noch langfristige Sicherungen durch Langzeitanstellung oder Sozialleistungen wie Pensionen vorgesehen. Das Aufrücken männlicher Dauerbeschäftigter, der »frühen Formen der Stammarbeiter« - in bezug auf soziale Sicherung, Ausbildung und betrieblichen Aufstieg - kontrastierte mit der »Sackgassensituation《 der meisten Industriearbeiterinnen. Der Marxist Ouchi wies darauf hin, daß Verbesserungen sich in den führenden Arbeiterschichten stärker oder gleich durchschlugen; die Differenzierung zwischen den fragmentierten Gruppen blieb also erhalten. Er verglich diese Struktur des »Aufwärtsrückens « der fragmentierten männlichen Belegschaften mit dem Bild einer Rolltreppe, bei der sich das gesamte Stufengefälle gleichzeitig bewegt. Die Industriearbeiterinnen hatten an diesem stufenweisen Aufstieg keinen Platz, sie bildeten die Grundebene, für die relativ einheitliche - schlechte - Bedingungen galten (zit. nach Lenz 1984: 228).

Diese Diskriminierung wurde begründet mit der patriarchalischen Arbeitsteilung und Hierarchie in den meist bäuerlichen Herkunftshaushalten. Während der männliche Haushalts vorstand Autorität über die Arbeiterinnen (und Teile ihres Lohns) beanspruchte, wurde der niedrige Frauenlohn mit dem Klassischen Argument des weiblichen »Zuverdienstes« zum Haushaltseinkommen legitimiert. Dem widersprach die Realität insofern, als Frauen, besonders die jungen Schwiegertöchter, wichtige Arbeitskräfte der bäuerlichen Hauswirtschaften waren und die Lohnsummen der Arbeiterinnen in der Regel erst den Fortbestand dieser Kleinwirtschaften ermöglichten (vgl. Lenz 1984). Es handelte sich dabei nicht um ökonomische »Nebenrollen «, wie es im Wort vom »Zuverdienst « anklingt, sondern um eine Abwertung der weiblichen Arbeitsleistungen, die ihren realen Beitrag verschleierte. Die soziale Unterordnung der Töchter und jungen Frauen im patriarchalischen »Haus« schlug sich also auch auf dem Arbeitsmarkt in Diskriminierung nieder. 
Die zweite Säule der Diskriminierung war eine neue geschlechtliche Polarisierung von Arbeitsbereichen und Zukunftschancen, die die im Industrialisierungsprozeß einsetzende Trennung von Heim und Betrieb, Reproduktion und Produktion begleitete. Wurde in der bäuerlichen Wirtschaft der zentrale Beitrag der Frau zum Haushaltseinkommen anerkannt, gehörte sie wie der Mann zu den »Arbeitenden«, so baute die kapitalistisch-industrielle Entwicklung langzeitlich auf einer Geschlechterpolarisierung der Bereiche Heim und Betrieb auf: Der Betrieb wurde - ungeachtet des relativ hohen Anteils weiblicher Beschäftigter - als eigentlicher Bereich der Männer betrachtet; das Heim wurde zum Bereich, später auch zum »Reich « der Frauen. Sie leisten fast alle Hausarbeit, treffen aber auch fast alle Entscheidungen im Haushalt selbständig (vgl. Lenz 1981). Die Polarität in der gesamtgesellschaftlichen geschlechtlichen Arbeitsteilung stützt sich auf die Weiterführung und Zuspitzung der gegenseitigen Ergänzung (Komplementarität) männlicher und weiblicher Àrbeitsfelder aus der bäuerlichen Welt, die in der nachholenden Entwicklung Japans ausgebaut wurde, und auf die materielle Anerkennung und rudimentäre Absicherung der Hausarbeit (s.u.). Schließ 3 lich ermöglicht sie auch die ungeheure Konzentration und Zuwendung der männlichen Stammbelegschaft auf den Betrieb, da die Hausarbeit und damit die Reproduktion der Arbeitskräfte allein von den Hausfrauen in ihrem »weiblichen Reich« geleistet wird. Diese Polarität und Komplementarität in der geschlechtlichen Arbeitsteilung enthält ein kompliziertes Wechselverhältnis von abstrakter Gleichheit und realer Ungleichheit: die Arbeitsbereiche »Heim« und »Betrieb《 erscheinen abstrakt gleichwertig und überdies die Hausarbeit als anerkannte, selbständige Tätigkeit. Doch wenn die Frauen ihr - im Denken der Geschlechtspolarität — 》gleichwertiges Heim《 verlassen, stoßen sie in Politik und Gesellschaft, vor allem aber im Bereich der Lohnarbeit auf massive Ungleichheit. So erweist sich die Geschlechterpolarität ${ }^{6}$ als modernisierte Stufe des Patriarchats, bei der die unmittelbare patriarchalische Herrschaft des Haushaltsvorstands durch einen generellen Sexismus ersetzt wurde. Ich möchte zunächst einige Unterschiede in der Entwicklung der Haushalte in Japan und Europa während der Industrialisierung berühren, die erklären, warum die japanischen Hausfrauen das Heim so lange als »ihren weiblichen Ort « sahen. Danach möchte ich auf die Konsequenzen der Geschlechterpolarität in der betrieblichen Arbeitspolitik zu sprechen kommen.

Die Vorstellung der Komplementarität der geschlechtspolaren Arbeitsteilung bedeutete vor dem Hintergrund der bäuerlichen Arbeitsteilung, daß der Haushalt im öffentlichen Bewußtsein und im Selbstgefühl der Frauen seine ökonomische Bedeutung nicht verlor. Hausarbeit wurde weiterhin als gesellschaftlich nützlich angesehen und bereits um 1960 wurde in der $\gg$ Hausfrauen-Debatte ( (shufu ronso) die Forderung nach ihrer Entlohnung erhoben (Ueno 1982). In Japan sprach man von einem qualitativen Wandel in der Familienarbeit, der durch den Rückgang der Kinderzahl und der »häuslichen Eigenproduktion «, aber auch durch wachsende qualitative Anforderungen gekennzeichnet sei - und nicht wie in Deutschland von einem »Funktionsverlust der Familie«. Die in Europa nachgezeichnete Transformation von »Arbeit in Liebe« fand in der japanischen Industrialisierung nicht statt.

Die Hausarbeit der Frau hat zudem eine, wenn auch prekäre materielle Absicherung durch den Gewohnheitsanspruch der Hausfrauen, die Lohntüten ihrer Männner ganz oder größ- 
tenteils zu erhalten. Für den Haushaltskonsum können sie das Einkommen des Mannes beanspruchen und selbständig verwalten; das Feilschen oder »Betteln« ums Haushaltsgeld gibt es in den japanischen Lohnarbeiterfamilien nicht. Allerdings kann dieser Brauch unterschiedlich bewertet werden: Drückt sich darin einerseits ein ökonomischer Anspruch der »hauswirtschaftlichen « Ehefrau aus, so kann er andererseits so verstanden werden, daß der Mann »seine Frau und Familie« ernährt (ibid. 1982: 7 ff.).

Parallel zur industriellen Expansion in der Zeit des »hohen Wachstums « nach 1955 wurde die Kernfamilie anstelle des Mehrgenerationenhaushaltes, der stark von der $i e$ Tradition geprägt war, zur führenden Familienform. Mit ihr verband sich eine Suche nach dem »Glück daheim« (»My home-ismus«) und ein Machtrückgang der älteren Generation. Die Machtposition der Hausfrauen wurde durch den stufenweisen Rückzug der männlichen Autoritäten gestärkt: die Schwiegerväter/Haushaltsvorstände hatten durch Rechtsreformen und die Ausbreitung der separat lebenden Kleinfamilien ihre vorige dominante Stellung eingebüßt. Die Ehemänner wurden in dem wirtschaftlichen Aufschwung in die Betriebe »eingezogen « und waren im Haushalt nur noch wenig präsent. Das inverse »Küchenmatriarchat « der japanischen Kernfamilie und der relativ eigenständige Entscheidungsspielraum der japanischen Hausfrauen ist also auch dem »Ausfall der Väter« zu verdanken. Mit der alleinigen Verantwortung der Frau für Hausarbeit und alltägliche Erziehungsfragen wuchs jedoch ihre Belastung.

Anders als in Europa ist die zentrale Beziehung in der Kernfamilie die zwischen Mutter und Kindern und nicht das Gatten- oder Partnerverhältnis (vgl. Lenz 1981). Die Betonung der Mutterrolle, die das gegenwärtige Frauenbild bestimmt und sich auch in der gewerkschaftlichen Politik wiederspiegelt (vgl. Herold 1980), ist ein markantes Beispiel für die Verschüttung und Synthese der »Tradition« im Prozeß der Modernisierung: Auch in Japan kann von der »modernen Mutter und Hausfrau« gesprochen werden, auch wenn sich ihre Position und ihr Bewußtsein von ihrer »westlichen« Gegenspielerin unterscheidet. In den bäuerlichen Haushalten wurden die jungen Frauen vor allem auf ihren Beitrag als Produzentinnen festgelegt; Großeltern und ältere Geschwister teilten mit ihnen die Versorgung der Kleinkinder, trugen sie häufig tagsüber auf dem Rücken herum. Die Schwiegertochter im Bauernhaushalt konnte scheel angesehen werden, wenn sie sich viel um ihre Kinder kümmerte und nach Meinung der Schwiegermutter die Felder oder den Webstuhl vernachlässigte (vgl. Lenz 1984: 156-172). Im gegenwärtigen Bild der »traditionellen japanischen Frauenrolle als Hausfrau und Mutter « (vgl. Hirano 1984) ist die Tradition dieser weiblichen Stärke und Härte verschüttet. Einerseits bezieht es sich auf die Tradition der samurai und Kaufleute; andererseits wirkten häufig übersehene Einflüsse der frühen Frauenbewegung mit ihrem Kampf um freie Mutterschaft und der damaligen Massenkultur auf die Modernisierung der Mutterrolle ein.

Die Mutter-Kind-Beziehung wird von beiden Seiten mit starken Emotionen besetzt, während die Gattenbeziehung häufig als nüchternes Verhältnis von Ansprüchen und Versorgung erscheint. Das Kleinkind hält sich meist bei der Mutter auf, die auf seine Bedürfnisse unmittelbar eingeht. Nach Einschätzung des Psychologen Doi Takeo entsteht so ein Beziehungsmuster der $\gg$ Freiheit in Geborgenheit «, das auch die Beziehungsstrukturen im späteren Leben und zu Institutionen prägt: der Wunsch, »mütterlich« versorgt zu werden, sich 
frei zu fühlen, in dem man »aufgehoben« wird (vgl. Doi 1982). Die älteren Kinder sind in ihrer Erziehung und Versorgung stark von der Mutter abhängig, und die Belastungen für die Mütter, die ihre Kinder durch die »Examenshölle« des japanischen Schulsystems steuern müssen, haben zugenommen. Sowohl das herrschende Mutterbild als auch die reale Mutter-Kind-Beziehung binden die Frauen an Kinder und Familie.

Ich bin auf die Veränderungen des Haushalts eingegangen, um nachzuzeichnen, wie die Stellung der Frauen auf dem Arbeitsmarkt durch das doppelte Bezugssystem des Haushalts und der betrieblichen Politik bestimmt wird. Es läßt sich vermuten, daß die Festschreibung der weiblichen Beschäftigten im Bereich der Privatunternehmen auf den Sektor eher einfacher Tätigkeiten ohne Aufstiegschancen außerhalb der garantierten Dauerbeschäftigung durch die Betonung der Geschlechterpolarität konstant blieb. Doch veränderten sich die Zusammensetzung der weiblichen Beschäftigten und damit auch die Begründungen für ihre betriebliche Diskriminierung: Die Unterordnung der jungen Frauen - durch ihre Definition als Töchter des patriarchalischen Hauses bis 1945 - wurde abgelöst durch eine neue Festlegung und Eingrenzung der weiblichen beruflichen Chancen - über eine Betonung der Mutterrolle in der modernen Kernfamilie. Die Dialektik der Geschiechterpolarität in Haushalt und Lohnarbeit, die von scheinbarer Gleichheit der Sphären zu realer Ungleichheit führt, hat schon Oba Ayako 1969, auf dem Höhepunkt der industriellen Expansion während des »hohen Wirtschaftswachstums«, kritisiert und mit der Frage der privaten menschlichen Reproduktionsarbeit verbunden:

»Das Problem der Frauenarbeit ist nicht einfach eine Frage der Arbeitswelt; es hängt mit der Position der Frauen in Familie und Gesellschaft zusammen. In einer Gesellschaft, die Schwangerschaft, Geburt und Kindererziehung als individuelle Gegebenheiten der Frauen ansieht, sind gleiche Rechte und Pflichten von Mann und Frau in der Arbeit ein leeres Versprechen. Während in Japan heute die Gleichheit von Mann und Frau in Familie und Ausbildung verwirklicht scheint, lehrt ein Schritt in die Arbeitswelt, daß Mann und Frau nicht auf der gleichen Stufe stehen. Die Position der Arbeiterin, die nicht als vollwertige Arbeitskraft beurteilt wird, verweist auf eine schwache Stelle der Frau in der Familie« (Oba/Ujihara 1969: ii).

Haushalt und Betrieb bilden ein doppeltes Bezugssystem, das die sich wandelnde Stellung der Frauen auf dem Arbeitsmarkt beeinflußt: Einerseits wirkt sich die Festlegung der Frau auf »ihren Verantwortungsbereich Haushalt « auf ihre Zuordnung zu den Randbelegschaften aus. Andererseits wird die Geschlechterpolarität in betriebliche Regeln des differenziellen Zugangs zu sozialer Sicherung durch Garantien von Dauerbeschäftigung, Ausbildung und Aufstieg aufgenommen, in denen die Interessen des Managements an einer Integration und Kontrolle der Beschäftigten zum Ausdruck kommen.

Auf dem Fundament der Geschlechterpolarität baut das »Modell der japanischen industriellen Beziehungen « für die Kernbelegschaften mit seinen drei Säulen der Dauerbeschäftigung, des Senioritätslohnes und der Betriebsgewerkschaften auf. Deswegen enthält es in seinen wesentlichen Bestandteilen eine implizite oder explizite Diskriminierung der Frauen und auch anderer Gruppen außerhalb des Leitbilds der männlichen, dauerbeschäftigten, qualifizierten Arbeitskraft, wie z.B. der älteren Beschäftigten oder der Wanderarbeiter (vgl. Ernst 1980). Die betriebliche und staatliche Arbeitspolitik ging von dem Grundsatz aus, daß der Betrieb der Bereich der Männer und der Haushalt der der Frauen sei. Damit wurde das Modell der Dauerbeschäftigung männlicher Stammarbeiter, die Langzeitperspektive 
in ihrer betrieblichen Ausbildung und Aufstiegsleiter legitimiert und zugleich die Zuweisung der großen Mehrheit der Frauen zu den Arbeitsmarktsektoren begründet, die durch Kurzzeitperspektive, Instabilität und einfache Tätigkeiten gekennzeichnet sind. (Oba/Ujihara 1969). Diese Geschlechterpolarität auf dem Arbeitsmarkt ignoriert allerdings, daß Frauen auch nach der Eheschließung und vielleicht einer kurzen Pause zur Versorgung der Kleinkinder in wachsendem Maße weiterhin erwerbstätig sind. Da sie also faktisch ein langfristiges Beschäftigungsinteresse haben, ist diese Verkürzung ihrer Lohnarbeitsperspektive ideologisch, während ihre Zuordnung zum Haushalt einen materiellen Kern hat. Die hier angedeutete Fragmentierung der Belegschaften entlang der Geschlechtsgrenzen spiegelt sich auch auf der Ebene ihres Betriebsbewußtseins und ihrer internen »Grenzziehungen « bei der Definition von Gruppen- und Betriebsgemeinschaft wieder. Die Garantie der Dauerbeschäftigung richtet sich auf »Rundum-Nutzung der Arbeitskraft « der Kernbelegschaften (vgl. Deutschmann/Weber in diesem Heft) und auf die Herausbildung einer entsprechenden Betriebsloyalität. Zugleich kann das Management gegenüber den weiblichen Randbelegschaften z.B. bei Personalkürzungen auf ihre primäre Bindung an den Haushalt nach dem Motto »Die Familie ist das Glück der Frau« verweisen.

\section{Die Geschlechterpolarität in đer betrieblichen Arbeitspolitik}

Wie auch in anderen ostasiatischen Ländern (Malaysia, Indonesien), ist in Japan die Frauenerwerbsquote, hier mit ca. 50 \%, relativ hoch (Rodosho fujinkyoku 1984, Anhang 1). Während der $\gg z$ weiten Industrialisierungsphase « Japans, zur Zeit des hohen Wirtschaftswachstums 1955-1972, und zu Beginn der 1980er Jahre stieg die Zahl der weiblichen abhängigen Beschäftigten überdurchschnittlich an. Wir können von zwei aufeinanderfolgenden $\gg$ Beschäftigungswellen« von Frauen sprechen: Während des hohen Wirtschaftswachstums zog ein Teil der expandierenden Massenproduktions-Industrien, wie die Metall-, Elektround Lebensmittelindustrien, junge Frauen an, die Mittel-oder Oberschulabschlüsse hatten, teils vom Land kamen und in einfachen, repetitiven Tätigkeiten wie der Montage eingesetzt wurden. Zugleich strömten junge Männer in die wmännlich besetzten Bereiche« der Massenproduktion z.B. an die Fließbänder der Autoindustrie. Der häufig hervorgehobene Mangel an jungen Schulabgängern auf dem Arbeitsmarkt Ende der $60 \mathrm{er}$ Jahre, von dem stärkere horizontale Tendenzen auf dem Arbeitsmarkt und eine längerfristige Nivellierung der Bevorzugung der dauerbeschäftigten Kernbelegschaft erwartet wurden, führte auch zu einer starken Nachfrage nach jungen Frauen (Akamatsu 1969: 100); allmählich weiteten die Betriebe die Teilzeitbeschäftigung vor allem von Ehefrauen oder Müttern aus. Die zweite große Beschäftigungswelle schwemmte in den späten 1970ern Schulabgängerinnen und Universitätsabsolventinnen und Teilzeitbeschäftigte in den Dienstleistungssektor. Vor allem in Handel, Banken und Versicherung wurde diese vorqualifizierte weibliche Arbeitskraft für einfache und assistierende Tätigkeiten und für eine $» F$ Flexibilisierung « der Arbeitszeiten und Beschäftigungsformen eingesetzt; typisch dafür sind die »Assistentinnen « hinter den Bankschaltern, die Teilzeitarbeiterinnen in den großen Kaufhausketten wie die noch seltenen Heimarbeiterinnen an ihren Bildschirmgeräten. 
Tabelle 1: Sektorale Verteilung der weiblichen abhängig Beschäftigten (\%)

\begin{tabular}{lrrr} 
& 1960 & 1970 & 1980 \\
\hline Primärer Sektor & 2,3 & 0,9 & 0,7 \\
Sekundärer Sekior & 40,6 & 37,8 & 31,0 \\
Tertiärer Sektor & 56,5 & 61,2 & 68,0 \\
\hline
\end{tabular}

Quelle: Rodosho fujinkyoku 1985: fu 14

Tabelle 2: Beschäftigung in ausgewählten Berufsfeldern nach Geschlecht 1960-1984 (in tausend Personen)

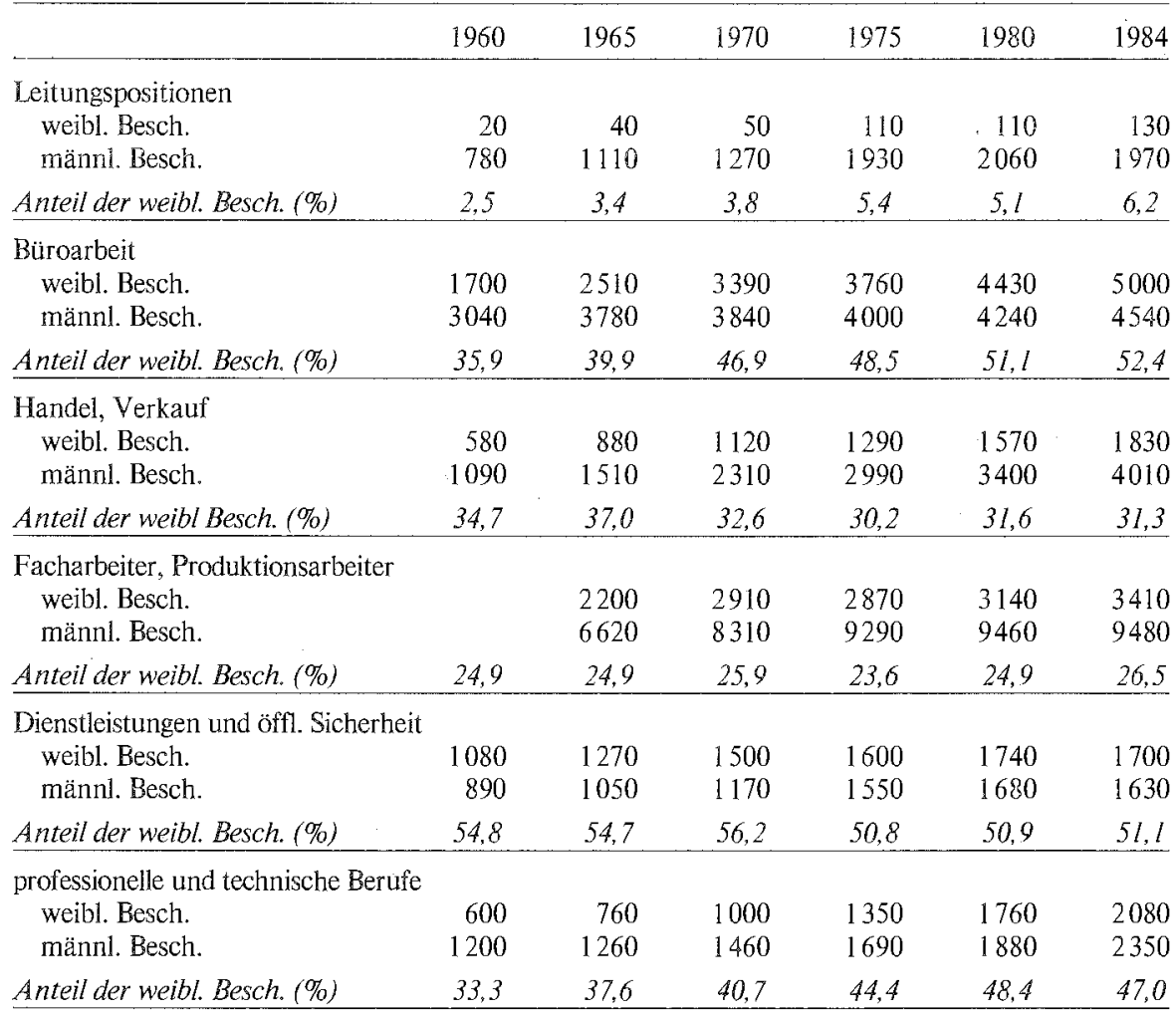

Quelle: nach ibid: fu $12-3$

Die Berufsstatistik zeigt mit $50 \%$ einen relativ hohen Frauenanteil in Büro, Dienstleistungen und technischen und professionellen Berufen; auch im Handel und in geringerem Umfang in der industriellen Produktion stellen Frauen ca. ein Drittel bzw. ein Viertel der Beschäftigten. 
Der stattliche Anteil in professionellen Berufen dürfte auf die Bedeutung von Frauen im Sozialbereich, teils in typischen Frauenberufen wie Kindergärtnerinnen und Krankenschwestern, sowie der Lehrerinnen zurückgehen. Kaum vertreten sind Frauen dagegen in den Leitungspositionen, was schon für unterste Ebenen gelten dürfte (vgl. Herold 1980), und den Branchen Verkehr, Transport und Kommunikation (Rodosho fujinkyoku a.a.O.).

In der Industrie wuchs die Frauenbeschäftigung rasch an; im Bereich von Handel und Dienstleistungen verdreifachte sie sich sogar - aber vor allem in den untersten Rängen. In Industrie, Handel und Dienstleistungen hatten Frauen überwiegend einfache und/oder untergeordnete »assistierende « Tätigkeiten, die niedriger entlohnt wurden (Oba/Ujihara 1969: 182 ff., 213-233). Ein »Ausbruch《 aus den untergeordneten Arbeitsmarktsektoren, wie ansatzweise in den USA und Europa geschehen, gelang ihnen aber nicht - trotz der gewaltigen Ausweitung weiblicher Beschäftigung in den 1960er und 70er Jahren und trotz des Anstiegs ihres allgemeinen Qualifikationsniveaus. Dies Verharren in der betrieblichen Ungleichheit ist angesichts des markanten wirtschaftlichen und sozialen Strukturwandels in Japan erklärungsbedürftig.

Eine Ursache dafür liegt in der Übernahme der Geschlechterpolarität in die industriellen Beziehungen der Großbetriebe, die über maßgeblichen gesellschaftlichen Einfluß verfügen. Ich will diesen Prozeß in der Folge am Beispiel der Beschäftigungssicherheit und der betrieblichen Ausbildungs- und Aufstiegssysteme kurz umreißen. Zuvor möchte ich allerdings anmerken, daß der Geschlechtsdifferenzierung in den Großbetrieben auch Kämpfe um Gleichheitsforderungen entgegenstanden, die vor allem im öffentlichen Dienst zu Erfolgen führten, aber auch von einem Teil der nationalen Gewerkschaftszentren, besonders in Sohyo und von Minderheitsgewerkschaften begonnen wurden.

\section{a) Zur Beschäftigungssicherheit}

Die Garantie der Dauerbeschäftigung, oft nicht ganz passend »lebenslange Beschäftigung « genannt ${ }^{7}$, ist wohl das im Westen bekannteste Merkmal der »japanischen industriellen Beziehungen«. Sie hatte Vorläufer in den 20er Jahren, verallgemeinerte sich aber erst in der industriellen Expansion der Nachkriegszeit. Die zugrundeliegende Rationalität könnte als »begrenzte Kooptation « der Kernbelegschaften verstanden werden, die nun ihre lebenslangen Perspektiven der Lohnarbeit und des sozialen Aufstiegs mit dem Wohl des Betriebs verbinden. Auch wenn sie sich mit emotionalen Momenten wie einer hohen betrieblichen Loyalität und dem »Betriebsgemeinschaftsdenken« koppelt, so lagert sie doch auf einem Kernbereich ökonomischer Interessen auf: Die Versprechen von Beschäftigungssicherheit und Beförderungen wurden während der Jahrzehnte der Wirtschaftsexpansion im wesentlichen eingelöst.

Nach der Niederlage der starken Gewerkschaftsbewegung der Nachkriegszeit erhielt diese begrenzte Kooptation mittels Dauerbeschäftigung allerdings einen deutlich partikularen Charakter. Als ihre Voraussetzung gilt das Wohlergehen des Betriebs, und damit dieser im Wettbewerb bestehen kann, muß er nach einem solchen Verständnis die unterschiedlichen Belegschaftsgruppen möglichst intensiv nutzen: Während die Betriebsgewerkschaften als Vertreter der Kernbelegschaften eine »lebende Mauer « um die Dauerbeschäftigung und ihre 
Vorteile bilden, halten sie sich bei dem Schutz der Randbelegschaften, besonders den Gruppen, die aus der Gewerkschaft ausgeschlossen sind (wie Teilzeitarbeiter/innen, Leiharbeiter/innen usw.) sehr zurück oder behandeln ihre Interessen insgesamt als unwichtig.

Frauen sind in der fragmentierten »Belegschaftspyramide« überwiegend an zwei Orten anzutreffen: am Rand der Kernbelegschaften oder in der wachsenden Schicht der instabil Beschäftigten, vor allem unter den Teilzeit- und Leiharbeiter/innen. Am Rand der Kernbelegschaften befinden sie sich deshalb, weil bei ihnen die Garantie der Dauerbeschäftigung relativiert oder gebrochen wurde (vgl. Nihon seisansei honbu 1985: 25 ff.). Parallel zur Ausweitung dieser Garantie für männliche Kernbelegschaften wurden betriebliche Regelungen zur zeitlichen Begrenzung und Befristung der Frauenbeschäftigung entwickelt: Diese Regelungen oder gar Abkommen mit der Betriebsgewerkschaft sahen ein betriebliches Ausscheiden der Frauen bei Heirat, Mutterschaft oder Erreichen von bestimmten Altersgrenzen (meist um 30-35 Jahre) vor. Geltenden Regeln, die nach einem Jahrzehnt juristischer Kämpfe, 1977 noch bei 7,4 \% aller Unternehmen bestanden (Herold 1980: 152), dürften die Spitze des Eisbergs entsprechener informeller betrieblicher Praktiken bilden. Laut Äußerungen des Managements wurde ab Mitte der 70er Jahre, im Zuge forcierter Rationalisierung und mikroelektronischer Automation, der Druck auf Arbeitsaufgabe verheirateter Frauen und Mütter, die an den aufgelösten Montagebändern gesessen hatten, aktualisiert (vgl. Lenz 1986). Erleichtert wurde diese Beschäftigungsanpassung durch die Einstellung vieler Frauen, während der Versorgung der Kleinkinder lieber im Haushalt zu bleiben, und durch die Zurückhaltung der Betriebsgewerkschaften (vgl. Herold 1980: 152-153).

Die staatliche Arbeitsmarkt- und Sozialpolitik nahm diese Zeitbegrenzungen in dem DreiPhasen-Schema eines (spezifisch) weiblichen Lebenszyklus auf, das die Beschäftigungspolitik der Betriebe mit beeinflußt haben dürfte. Nach Erwerbstätigkeit in der Jugend sollten Frauen eine Familien- und Kindererziehungsphass cinschieben und auf den Arbeitsmarkt zurückkehren, wenn die Kinder in die Schule kommen oder selbständig werden. Dies Modell wirkte auch in Europa auf die Arbeitsmarktpolitik ein; doch ein »Bruch « in der Betriebszugehörigkeit hat in Japan wegen des Leitmodells der Dauerbeschäftigung und des damit verbundenen Statuswechsels andere Konsequenzen. Die Dauerbeschäftigung gilt im wesentlichen für die direkt nach Abschluß der Ausbildung Eingestellten und nach ihnen für Betriebswechsler, aber sie erlischt nach Ausscheiden aus dem Betrieb in der Regel unwiderruflich. Mit dem Ausscheiden aus der Statusgruppe der regulär Beschäftigten sind die Frauen also auf weniger gesicherte Beschäftigungsverhältnisse in der Teilzeitarbeit oder in den Klein- und Mittelbetrieben verwiesen. Häufig bleibt ihnen auch aufgrund der Öffnungszeiten der Kindergärten und-krippen keine andere Wahl.

Wenn die Dauerbeschäftigung also die Funktion hat, betriebliche Loyalität und umfassenden Einsatz von Kernbelegschaften zu verstärken, so stellt sich die Frage, wie das Management trotzdem Anreize für die tendenziell davon ausgeschlossenen Frauen entwickeln konnte, um beispielsweise an den Fließbändern der Massenprodultionsindustrien die bekannte »japanische Qualität « herzustellen? Hier kann ich nur kurz die wichtigsten Mechanismen von Integration und Kontrolle aufzählen: (1) Ein paternalistischer Managementstil (2) und die Einführung der betrieblichen Kleingruppen, die wie die Gruppen zur Qualitätskontrolle oder das $\gg$ Big-Sister Movement« zugleich den persönlichen Arbeitsstolz und die 
Gruppenidentität ansprechen - und so einander unterstützende und kontrollierende Netzwerke bilden. Schließlich bot das Management (3) aber auch Kompensationen durch unmittelbare Entlastungen in der Arbeitszeitpolitik: die Einführung der Fünf-Tage-Woche ging bspw. von dem Elektrokonzern Matsushita aus. Sie wurde von den Arbeiterinnen begrüßt, wenn sie auch feststellen mußten, daß restriktivere Regelungen beim Jahresurlaub und Menstruationsurlaub den »Zugewinn« relativierten (vgl. Oba/Ujihara 1969: 221 ff.; Deutschmann/Weber in diesem Heft).

Grundlegender dürfte aber (4) sich die Geschlechterpolarität im Bewußtsein eines Großteils der Frauen im Sinne einer Anpassung an die Ausgrenzung aus der Dauerbeschäftigung ausgewirkt haben - die immerhin während einer Phase akuter Knappheit an jungen weiblichen Arbeitskräften üblich wurde. Im Rahmen der gegebenen Betriebsgewerkschaft mußte ein Ummünzen ihrer starken Position auf dem Arbeitsmarkt auf strukturelle Hindernisse stoßen; zudem erschwerte ihre minimale Repräsentanz in den Führungsgremien der Gewerkschaftsverbände und Betriebsgewerkschaften (vgl. Herold 1980: 189-200; Tanaka/Hitaka 1969: 183-188) starke Proteste. Dennoch blieb die diskriminierende Regelung der Beschäftigungssicherheit nach Geschlecht nicht ohne Widerstand.

Ich kann hier nicht auf die langen Kämpfe von einzelnen Frauen - teils mit, teils ohne gewerkschaftliche Unterstützung - gegen die diskriminierenden Entlassungsregeln eingehen (vgl. dazu Cook, Hayashi 1980). Mit ihrem Bestehen auf dem Recht regulärer Beschäftigung zeigen diese Kämpfe, daß die Gleichheitsforderungen inmitten der Geschlechterpolarität Wurzeln gefaßt haben, ohne daß sie diese einstweilen unterminieren oder erschüttern könnten. Im Öffentlichen Dienst, vor allem im Schulbereich haben die relativ starken Gewerkschaftsverbände bereits in den 1950er Jahren die Voraussetzungen für kontinuierliche Erwerbsarbeit, erweiterten Mutterschutz und - bei den Lehrern - Lohngleichheir erkämpft (Tanaka, Hitaka 1969: 190). Auf informeller Ebene setzen sich in den letzten Jahren weibliche Beschäf̣tigte mit ihrem Verhalten für ihre Dauerbeschäftigung ein: Ihre durchschnittliche Beschäftigungsdauer ist von 3,9 Jahren 1965 auf 6,3 Jahre 1983 angestiegen und ein Anteil von ca. $20 \%$ hat die Zehnjahresgrenze der Betriebszugehörigkeit überschritten (Akamatsu 1985: 9).

Die Teilzeitarbeit hat sich von 1960-1984 in etwa verdreifacht und wurde zum größten Feld instabiler Beschäftigungsverhältnisse mit niedriger Entlohnung und ohne soziale Sicherung; mit einem steigenden Frauenanteil von $42,8 \%$ im Jahre 1960 auf $70,6 \%$ im Jahre 1984 wurde sie während dieser Zeit »feminisiert« (vgl. Tabelle 3).

Tabelle 3: Zunahme der Kurzarbeit 1960-1984 (in Mio. Personen)

\begin{tabular}{lccccc}
\hline & 1960 & 1970 & 1975 & 1980 & 1984 \\
\hline Zahl der Kurzzeitbesch. & 1,33 & 2,16 & 3,53 & 3,90 & 4,64 \\
Anteil an abhängig Besch. (\%) & 6,3 & 6,7 & 9,9 & 10,0 & 11,1 \\
Zahl der weibl. Kurzzeitbesch. & 0,57 & 1,30 & 1,98 & 2,56 & 3,28 \\
Anteil an weibl. abh. Besch. (\%) & 8,9 & 12,2 & 17,4 & 19,3 & 22,1 \\
\hline
\end{tabular}

Quelle: Rodosho fujinkyoku 1985: fu 27 
In Industrie und Dienstleistungen ist der Anteil der Teilzeitarbeiterinnen an den weiblichen Beschäftigten stetig, in Handel und Verkauf spektakulär angestiegen (vgl. Tabelle 4). Diese Entwicklung hängt mit der »Fiexibilisierung« der Wirtschaft, der fortschreitenden Automation und dem wachsenden Gewicht des tertiären Sektors zusammen (vgl. Lenz 1987).

Tabelle 4: Verteilung der Kurzzeitarbeiterinnen (KZA) über ausgewählte Branchen (in 1000 Personen)

\begin{tabular}{lcccc} 
& 1975 & 1980 & 1982 & 1984 \\
\hline Prod. Industrie & 560 & 650 & 670 & 770 \\
Anteil an KZA $(\%)$ & 28,3 & 25,4 & 23,6 & 23,5 \\
Anteil der KZA an & 15,8 & 17,1 & 17,3 & 18,5 \\
weibl. abh. Besch. (\%) & 550 & 840 & 980 & 1180 \\
\hline Handel, Verkauf & 27,8 & 32,8 & 34,5 & 36,0 \\
Anteil an KZA $(\%)$ & 19,2 & 24,2 & 26,5 & 29,6 \\
Anteil der KZA an & 530 & 690 & 780 & 900 \\
weibl. abh. Besch. (\%) & 26,8 & 27,0 & 27,5 & 27,4 \\
\hline Dienstleistungen & 17,4 & 18,2 & 19,3 & 20,3 \\
Anteil an KZA $(\%)$ & & & & \\
Anteil der KZA an & & & & \\
weibl. abh. Besch. $(\%)$ & & & & \\
\hline
\end{tabular}

Quelle: Rodosho fujinkyoku 1985: fu 28

Da hier nur einige ausgewählte Branchen dargestellt werden, ergibt die Summe des Anteils der jeweiligen Branche an den KZA nicht $100 \%$.

Läßt sich die Ausweitung der Lohnarbeit für Mütter und Hausfrauen, gerade in der Form der Teilzeitarbeit, als kleiner Fortschritt für die Frauen interpretieren? Die Erwerbsquote der Ehefrauen lag schon 1952 bei $51,1 \%$, fiel dann bis 1975 auf $45,2 \%$ und stieg im Jahr 1984 wieder auf 53,6\% an (Rodosho fujinkyoku 1985:22). Ähnlich wie in Westeuropa und den USA drängen hauptsächlich Hausfrauen, häufig mit Kindern, in die Teilzeitarbeit. So können sie die alleinige Verantwortung für Hausarbeit und Kinder mit eigener Erwerbstätigkeit kombinieren. Aber schon die Kinderversorgung zeigt, unter welchem sozialen Druck sie beide Bereiche balancieren: Kindergärten sind i.a. nur 8 Std. geöffnet; da diese Zeit nicht ausreicht, um die Kinder rechtzeitig hinzubringen und abzuholen und einen Vollzeitarbeitsplatz — evtl. noch mit Überstunden — auszufüllen, bleibt nur die $»$ Entscheidung « für die Teilzeitarbeit. ${ }^{8}$

»Fortschritt《 hat seinen Preis - im Fall der Teilzeitarbeit die Abdrängung in einen Status weitgehend ungeschützter, niedrig entlohnter und einfacher Beschäftigung. Denn der Begriff der Teilzeitarbeit (paato taimu) bezeichnet in Japan nicht primär eine zeitliche Festlegung, sondern einen Beschäftigtenstatus. Dies zeigen einerseits die langen Arbeitszeiten: Mit einer durchschnittlichen Wochenarbeitszeit von 32 Std. und einem Arbeitstag von 6-7 Std. in 37,2\% der Betriebe und von 7-8 Std. in 33,2\% der Betriebe ist der Abstand zur Vollzeitarbeir nicht mehr groß, läßt man deren Überstunden-Verpflichtungen außer Acht. An- 
dererseits ist der Übergang von der Teilzeitarbeit zur regulären Vollbeschäftigung in der Regel verschlossen; die »Statusgruppen «sind gegeneinander abgeschottet. Da die Betriebsgewerkschaften i.a. nur die regulär Beschäftigten umfassen und Teilzeitarbeiter/innen wie andere instabil beschäftigte Gruppen nicht beitreten können, wird diese Abschottung in der Struktur der betrieblichen Interessenvertretung reproduziert. Die Beschäftigungsgarantie entfällt für die Teilzeitarbeiter/innen, wenn auch im letzten Jahrzehnt formale rechtliche Ansprüche wie Urlaub, Festlegung des Arbeitstags, Pausen stärker durchgesetzt wurden. Die Löhne liegen ein Viertel unter den ohnehin niedrigen durchschnittlichen Frauenlöhnen; häufig orientieren sie sich an den regionalen Mindestlöhnen (ibid).

Die Unternehmensinteressen an der Teilzeitarbeit lassen sich nicht auf die Funktion eines »Beschäftigungspuffers« reduzieren. Vielmehr ermöglicht deren ungeschützter Status einen regulären Einsatz bei einfachen Tätigkeiten zu niedrigen Löhnen, je nach den aktuellen betrieblichen Anforderungen. Auf eine Umfrage des Arbeitsministeriums gaben zahlreiche Unternehmen als Gründe für Teilzeitbeschäftigung an: Die durch Automation vereinfachten Arbeitsinhalte, die nun auch von Teilzeitarbeitern bewältigt werden können; die dadurch mögliche Senkung der Personalkosten und die Flexibilisierung der Arbeitsorganisation, z.B. durch einen Personaleinsatz nach Arbeitsanfall (Rodosho fujinkyoku 1985: fu 43). Neben der Delegierung einfacher und belastender Arbeit an Niedriglohngruppen gibt es ein Interesse an flexibler Beschäftigungsplanung und Anpassung, die durch den raschen Strukturwandel und die Erfahrung der Krisenbewältigung im letzten Jahrzehnt zusätzliche Bedeutung erlangt hat. Da die Belegschaftsfragmentierung durch die »Geschlechtsgrenze « im Alltag und dem Ausfallen der Betriebsgewerkschaft vertieft wird, erweitert sich der betriebspolitische Spielraum des Managements.

\section{b) Zur betrieblichen Ausbildung}

Die innerbetriebliche Ausbildung spielt in den japanischen Großbetrieben eine zentrale Rolle: Sie soll allgemeine fachliche Kenntnisse, Orientierungsfähigkeit und Flexibilität und zugleich betriebliche Loyalität, die »Firmenmorak vermitteln. In der Ausbildungsphase geschieht dies durch den Finsatz an verschiedenen Arbeitsplätzen und Abteilungen: Die Betriebe sind an einer vielseitigen, umfassend und flexibel einsatzfähigen und loyalen »Kerngruppe« von Stammarbeitern interessiert, nicht an spezialisierten Fachkräften. Ihr Vorteil liegt in einem größ3eren Handlungsspielraum bei der Zuweisung zu verschiedenen Arbeitsplätzen, der nicht wie in Europa dadurch begrenzt wird, daß die Beschäftigten sich auf ihre spezifische Qualifikation oder Arbeitsplatzstruktur berufen können. Für die Stammarbeiter bildet umgekehrt die betriebliche Ausbildung das Eingangstor zum allmählichen Aufstieg — vor dem Hintergrund der Grundsätze von Dauerbeschäftigung und Seniorität, die durch die Einführung von Leistungskriterien zwar modifiziert, aber nicht aufgehoben wurden.

Da die Betriebe mit der Ausbildung langfristige Interessen umfassender Nutzung der Arbeitskraft verbinden, gewährten sie sie zunächst, entsprechend dem Konzept der Geschlechterpolarität, nur den männlichen Kernbelegschaften. Um 1970 blieben auch Frauen mit Universitätsabschllüssen ebenso wie Bandarbeiterinnen vor diesem Eingangstor zum Aufstieg stehen. In der Elektroindustrie z.B. konnten die Frauen sich nicht an der Rotation 
durch verschiedene Abteilungen beteiligen, sondern blieben in ihren Montagegruppen. Selbst in »reinen Frauenarbeitsbereichen « konnten sie höchsten bis zur 》Gruppenführerin« aufsteigen während alle weiterführenden Positionen, vom Vorarbeiter bis zum Abteilungsleiter, männlich besetzt waren (Oba/Ujihara 1969: 232 ff.). Akamatsu stellte 1969 fest, daß Diskriminierung bei der Ausbildung, der Zuweisung der Arbeitsplätze, der Lohnerhöhung und der Beförderung die Regel sei. Da die Betriebe langzeitliche Beschäftigung nur für männliche Stammarbeiter vorsahen, betrachteten sie Investitionen in die Ausbildung von Frauen als sinnlos (ibid: 182).

Die Geschlechterpolarität in der Ausbildung brachte den Unternehmen den doppelten Vorteil, daß die »Geschlechtsgrenze« zwischen männlich besetzten untersten Leitungsfunktionen und Arbeiterinnen zur Kontrolle eingesetzt und die allgemeine, teils recht hohe »Vorqualifikation « der weiblichen Beschäftigten quasi unentgeltlich genutzt werden kann. Die Einstufung in »assistierende Tätigkeiten « Z.B. kann bedeuten, daß Universitätsabsolventinnen im Büro während der ersten Berufsjahre grundsätzlich die gleiche Arbeit machen wie ihre männlichen Kollegen, daneben aber Tee servieren und geringer entlohnt werden. Durch das Drängen von Frauen auf Zugang zu betrieblicher Ausbildung und Aufstieg, das in der Dekade der Frau große öffentliche Resonanz hervorrief, öffnete sich das Eingangstor ein Stück für die »Career women« mit hoher Motivation. Etwa die Hälfte der Betriebe sehen heute bei Neueinstellungen nach dem Abschlußexamen gleiche Bedingungen für Mann und Frau vor; bei Betriebswechslern fällt dieser Anteil auf ein Drittel. Ca. $80 \%$ bieten beiden Geschlechtern die gleiche Grundausbildung; $57 \%$ garantieren Chancengleichheit bei berufsbezogener Weiterbildung und ein Viertel beteiligt Frauen gleich an Ausbildungsschritten, die ejnen Aufstieg in untere Leitungsebenen ermöglichen (Rodosho fujinkyoku 1985: 14-16). Das Gesetz zur Chancengleichheit von Mann und Frau in der Beschäftigung aus dem Jáhre 1985 schreibt Chancengleichheit in den betrieblichen Ausbildungs- und Aufstiegssystemen vor, ohne aber Sanktionen bei Verletzungen bereitzustellen. ${ }^{9}$

Den Beschränkungen in der japanischen betrieblichen Ausbildung entspricht in Europa die notorische Schwierigkeit für Frauen, in qualifizierte technisch-gewerbliche Berufe vorzudringen. Aber die Verknüpfung mit dem System der Dauerbeschäftigung im Betrieb scheint in Japan die institutionellen Sperren zu erhöhen. Die Unternehmen haben sich in der Mehrheit nicht von dem Leitsatz gelöst, daß betriebliche Ausbildungsinvestitionen sich vor allem bei der männlichen Kernbelegschaft lohnen. Ein »Durchbruch « zeichnet sich in zwei neuen Bereichen ab, die beide unter Mangel an qualifizierten und hochmotivierten Arbeitskräften leiden: bei Kleinbetrieben im Bereich von Zukunftstechnologien und in der Programmierbranche, in der die Betriebe Frauen ausbilden und zugleich Modelle der Vereinbarung von längeren Kindererziehungszeiten und Dauerbeschäftigung überlegen, damit ihre Investitionen von Nutzen sind. ${ }^{10}$

\section{c) Geschlechterpolarität im Arbeitsschutz}

Die Gesetze zum Frauenarbeits- und Mutterschutz haben geschlechtspolare Vorstellungen übernommen. Zwar sehen sie bei Frauen einen Sonderschutz vor allgemeinen Belastungen vor, wie etwa in der Beschränkung von Überstunden, doch generelle Regelungen fehlen 
oder sind lückenhaft; zum Teil haben sie auch den Mutterschutzgedanken in dem Sinne erweitert, daß der Schutz des weiblichen Körpers und seiner Rhythmen berücksichtigt wurde. Der in den 1930ern eingeführte und im Arbeitsstandardgesetz 1947 verankerte Menstruationsurlaub wurde herkömmlicherweise mit dem Mutterschutz begründet (vgl. Herold 1980: 84-98). In dem jahrzehntelangen Eintreten der Frauen für seine Erhaltung sehe ich ein Einklagen der »weiblichen Differenz«, das in seinen Folgen ambivalent ist. Verweigern sich die Frauen unter dem Leitbild der (zukünftigen) Mutter der Abstraktion des Lohnarbeitsverhältnisses und betonen ihr Geschlecht, so trägt dieser Sonderschutz zugleich zur Verfestigung eines Sonderstatus für Frauen und eines entsprechenden Bewußtseins bei. Jahrzehntelang war der Menstruationsurlaub Ausgangs- und Angelpunkt in den Kämpfen. um Frauenrechte im Betrieb und der gewerkschaftlichen Bildungsarbeit für Frauen, während die hier umrissene systematische Diskriminierung in den Hintergrund trat (ibid; Oba/ Ujihara 1969: 234 ff.). Im 1985er Gesetz zur Chancengleichheit von Mann und Frau wurden die Sonderschutzregelungen bis auf den Mutterschutz im engen Sinne relativiert oder aufgehoben.

\section{Der arbeitspolitische Nutzen des Managements an der Geschlechterpolarität}

Ich will nun kurz die arbeitspolitischen Vorteile zusammenfassen, die dem Management aus der Inkorporation der Geschlechterpolarität mit ihrem doppelten Bezugssystem zwischen Betrieb und Haushalt für die industriellen Beziehungen erwuchsen. Dabei ist es notwendig, die Arbeitsteilung zwischen Heim und Betrieb und also die Reproduktionsarbeit der Hausfrauen miteinzubeziehen.

Auf die männliche Kernbelegschaft wirkt sich die Geschlechterpolarität im doppelten Sinne aus: Ihre Abkoppelung von der Hausarbeit, die allein den Hausfrauen überlassen wird, ermöglicht, zusammen mit den betrieblichen Faktoren, eine uneingeschränkte Verfügbarkeit im Sinne einer umfassenden Nutzung ihrer Arbeitskraft. Zum zweiten verstärkt die Geschlechtsgrenze zu dem weiblichen Teil der Randbelegschaften die Belegschaftsfragmentierung, was in einer partikularen Interessenvertretung mit Konzentration auf männliche Kernbelegschaften zum Tragen kommt. In einer Periode rascher Umstrukturierung und Flexibilisierung der Belegschaften gerät diese partikulare Politik, die in der Expansionsphase durchaus Erfolge verbuchen konnte, in die Defensive (vgl. Lenz 1987b).

Die Geschlechterpolarität in den industriellen Beziehungen garantiert zugleich den Fortbestand der geschlechtlichen Spaltung des Arbeitsmarkts und eine stetige Reproduktion der ungesicherten, untergeordneten Sektoren. Vor der Norm der langfristigen ununterbrochenen Betriebszugehörigkeit erscheint die Phase der Kinderversorgung und Familienarbeit der Frauen als »Bruch «, der sie in sekundäre Positionen entläßt. Dies gilt besonders dann, wenn Hausfrauen und Mütter auf den Arbeitsmarkł zurückkehren und in die Sackgasse der Teilzeitarbeit oder in $\mathbb{K}$ lein- und Mittelbetriebe abgedrängt werden. In den neueren Strategien der »Flexibilisierung« wird die Beschäftigung dieser wenig gesicherten Gruppen zu diskriminierenden Bedingungen in unterschiedlichen Kombinationen ausgeweitet (Lenz 1987b).

Selbstverständlich manifestiert sich die Geschlechterpolarität nicht allein in betrieblichen Regelungen; sie ist ein wichtiger Teil der alltäglichen Interaktionsbeziehungen, die am Ar- 
beitsplatz Konflikte und Konsens vermitteln (vgl. Burawoy 1985). Während die Partnerbeziehung oder Paarbeziehung in Japan nicht denselben sinnstiftenden Charakter hat wie in Europa, können geschlechtshomogene »Männer- oder Frauengruppen «ein festes Gemeinschaftsgefühl entwickeln. Bei der Gruppenbildung in der männlichen Kernbelegschaft überlagert es sich mit dem sorgfältig geförderten Betriebsgemeinschaftsbewußtsein; dazu kommen männlich gefärbtes Arbeitsethos und Arbeitsstolz, etwa in dem Sinne »wir Kerle tragen mit unserer harten Arbeit und unserem Einsatz den Erfolg des Betriebs «. Dieses Arbeitsethos und die Gruppensolidarität begründen ein starkes kollektives Selbstbewußtsein, auf das gewerkschaftliche Mobilisierungsanstrengungen stellenweise zurückgreifen konnten. ${ }^{13}$ Doch im Alltag wenden sich beide Orientierungen »nach innen «, zur Arbeitsgruppe oder männlichen Kernbelegschaft. Das Gemeinschaftsgefühl wird durch informelle männerzentrierte Institutionen, wie die gemeinsamen Trinkabende, bei denen Kolleginnen »Fremdkörper« wären, verstärkt. Es verbindet sich mit einer deutlichen Abgrenzung zu »denen draußen « - den Teilzeitarbeiter/innen, Leiharbeiter/innen, Beschäftigten in anderen Betrieben. Während die Kolleginnen wohl als Mitglieder der Betriebsgemeinschaft gesehen werden, kann die geschlechtshomogene Männergruppe sie nicht integrieren; sie sind »drinnen und draußen « zugleich.

Die Frauengruppen in der Produktion oder in der Kleingruppenbewegung könnten ähnliche Funktionen für die Bildung von alltäglichem Konsens haben. Allerdings wird von ihnen wohl kein Betriebsgemeinschaftsgefühl erwartet, eher eine gegenseitige Anleitung wie im »Big Sister Movement«, bei dem länger beschäftigte Arbeiterinnen die jüngeren unter ihre kontrollierenden Fittiche nehmen, oder eine Verstärkung der Arbeitsmotivation wie in den Qualitäts-Kontroll-Gruppen. Beispielsweise sind in der Mikrochipfertigung mit ihren hohen Qualitätsanforderungen und Belastungen Qualitätszirkel mit »weiblichen Namen«, wie etwa »Pink Ladies«, recht verbreitet (Lenz 1987a).

Es wäre zu fragen, ob die Unterschiede in den geschlechtsspezifischen betrieblichen Regelungen zwischen Japan und der Bundesrepublik nicht eher graduelle sind. Immerhin kennen wir auch Ausbildungs- und Aufstiegssperren für Frauen, die teils mit dem geschlechtspolaren Argument »sie heiratet ja doch « begründet wurden, oder die Unterrepräsentation von Frauen in der gewerkschaftlichen Politik. Möglich wäre auch, daß sich aufgrund der beruflichen Ansprüche junger Frauen in Japan und der Erosion des bestehenden Modells industrieiler Beziehungen eine Tendenz zur Konvergenz abzeichnet. Dazu will ich hier keine Prognose wagen. Als Resultat der nachholenden Entwicklung Japans, die auch die Geschlechterpolarität instrumentalisierte, läßt sich jedoch eine umfassende Mobilisierung der Beschäftigten in diesem Prozeß 3 und eine grundlegende Schwäche von horizontalen Gleichheitsnormen oder - bewegungen erkennen, die die starren und teils brüchig gewordenden Regelungen und Abgrenzungen hinterfragen und neue egalisierende Modelle vorschlagen könnten. Die beschriebene Verkürzung des Gleichheitspostulats und die Ineffektivität horizontal vermitteInder Instanzen haben zur Folge, daß das Feld sozialer Auseinandersetzungen für das Erreichen von Kompromissen oder grundlegenden sozialen Veränderungen anders strukturiert wird.

Diese andere Struktur der sozialen Auseinandersetzung führt, im Gegensatz zur äußeren Flexibilität der sozialen und ökonomischen Organisation in Japan, zu einer gewissen Starre. 
Sie erschwert die offensive Aufnahme neuer Entwicklungen und noch mehr die Entwicklung vorwärtsgewandter Strategien, die auch die große Mehrheit der Beschäftigten außerhalb der Kernbelegschaften, die Hausfrauen und andere Gruppen, die gegenwärtig die »Infrastruktur des japanischen Erfolgsmodells« aufrechterhalten, umfassen könnten. Denn das hier skizzierte Regulationsmodell zeigt auf drei Ebenen Fissuren und Brüche, die auf tiefergehende Bewegungen hinweisen: Zunächst steht die Konzentration der Betriebsgewerkschaften auf die Kernbelegschaften unter dem Druck komplexer Veränderungen: Die Kernbelegschaften und damit ihre Mitgliederzahlen werden durch den zunehmenden Einsatz von peripheren, ungesicherteren Beschäftigungsgruppen, darunter viele Frauen (Teilzeitarbeiter/innen, Leiharbeit/innen), allmählich erodiert; der Schutz selbst der zentralen Gruppe der männlichen älteren Stammarbeiter wird dabei fraglich (vgl. Lenz 1987b). Während die Betriebsgewerkschaften für ihre Trägerschicht offensive Konzepte wie eine dem technischen Wandel angepaßte Ausbildung vorschlagen, gelang ihnen bisher eine horizontale Umorientierung zu den instabil Beschäftigter und den Beschäftigten der ihren Betrieben zugeordneten Klein- und Mittelbetriebe nicht - was dem Schwinden ihrer sozialen Basis entgegenwirken könnte. Eine Lösung der Krise des betriebsgewerkschaftlichen Partikularismus ist nicht abzusehen.

Zum zweiten hat sich mit steigender Ausbildung, sinkender Kinderzahl und unter dem Einfluß der neuen Frauenbewegung die Einstellung gerade junger Frauen zur Lohnarbeit geändert. Das Interesse an langfristiger Beschäftigung und ihrer Absicherung wächst ebenso wie der Wunsch nach inhaltlich interessanter Arbeit jenseits der einfachen, untergeordneten oder als »assistierend« eingestuften Tätigkeiten, für die Frauen gegenwärtig vornehmlich eingesetzt werden (Nihon seisansei honbu 1985). Die ideologische Seite der Geschlechterpolarität, nämlich die Verleugnung der langfristigen weiblichen Erwerbstätigkeit, verliert dadurch ihre bisherige Ausstrahlung und ihre Funktion, den betrieblichen Konsens auf Basis der Unterordnung von Frauen zu stabilisieren, wird brüchig. SchlieBlich stöBt auch der Rückzug der Väter vom Haushalt zugunsten ihrer betrieblichen Karriere, der ja durch die umfassende Nutzung der Arbeitskraft der männlichen Kernbelegschaft (vgl. Deutschmann/Weber in diesem Heft) und die Konkurrenz im Betrieb forciert wurde, allmählich an die Grenze der Geduld eines Teils der Hausfrauen und Mütter. Zwar wird die alleinige Verantwortung für den Haushalt noch wenig angezweifelt; wohl aber wird die Belastung der Frauen in allen prakt ischen Fragen des Alltags, von der Erziehung bis zu kommunalen Angelegenheiten zunehmend kritisch hinterfragt. ${ }^{12}$

Die Tendenzen zur $» J a p a n i s i e r u n g «$ stoßen in der Bundesrepublik auf eine gleich heitlichere Tendenz, ${ }^{13}$ können diese aber unter vermeintlich geschlechtsneutralen Leitworten wie $\gg \mathrm{Ef}$ fektivität und Leistung von Spitzenbelegschaften« durchaus modifizieren. Eine wachsende Partikularisierung, die Herausbildung von verschieden stark abgesicherten Gruppen auf dem Arbeitsmarkt, die viele Frauen umfassen, stellt die »R ückseite« der japanischen Verhältnisse dar. Wenn in dieser Situation in der Frauenfrage »eine Politik des Unterschieds« (Erler 1985) vorgeschlagen wird, so könnte die Auseinandersetzung mit einer Industriege sellschaft durchaus lohnen, in der jenseits von vorindustriellen Projektionen diese Politik systematisch inkorporiert wurde. Zugleich kann dieser Vergleich zeigen, daß Gleichheit zwar viel, aber keineswegs alles ist. 


\section{Anmerkungen}

1 Dies erscheint mir besonders wichtig angesichts der Bagatellisierung von Forderungen der bürgerlichen Revolution wie Freiheit und Gleichheit in Ansätzen der Frauenforschung, die die Geschlechtsdifferenz betonen und eine uniforme kapitalistische Entwicklung, die die $\gg$ Hausfrauisierung « notwendig zur Grundlage hat, annehmen. Dem wird besonders bei Illich das Gegenbild intakter Geschlechtssphären in vorindustriellen Zeiten entgegengestellt (vgl. Illich 1984).

2 Ebenso interessant ist die Debatte um die Durchsetzung der Patriarchats in Japan: einem Teil der Autor/innen zufolge sind sowohl matriarchalische Strukturen als auch die Evolution zum Patriarchat auf Grundiage schriftlicher Quellen zu rekonstruieren.

3 Vgl. dazu auch die Diskussion über »the making of tradition« in Hobsbawn/Ranger 1983.

$4 \mathrm{Vgl}$. auch die Diskussion der innergewerkschaftlichen Demokratie bei Shirai 1982: 65 ff.; die Gleichheitsdebatte ist u.a. durch das Gesetz zur Chancengleichheit von Mann und Frau in der Beschäftigung (danjo koyo kikai kinto ho) (1985) belebt worden.

5 . Status ist hier also nicht im Sinne der Parsonschen Handlungstheorie zu verstehen.

6 Ich spreche von Geschlechterpolarität, wenn einem Geschlecht Arbeitsbereiche alleinig oder überwiegend zugeschrieben und diese Bereiche der Geschlechter gegeneinander abgeschottet werden.

7 Die Garantie der Dauerbeschäftigung wurde z.B. in den Personalreduktionen während der Rationalisierung der späten 1970er Jahre für ältere Beschäftigte relativiert, die mit einem »Schulterklopfen« (katatataki) in den Ruhestand gebeten oder entlassen wurden. »Lebenslange Beschäftigung« müßte aber auch für diese Gruppe gelten.

8 Neben der Infrastruktur der Kindergärten stellt die staatliche Steuerpolitik ein regulierendes Mo ment dar: Die Teilzeitarbeiterinnen versuchen, ihre Einkünfte unter dem Steuerfreibetrag für das Lohneinkommen des Ehepartners zu halten.

9 Das Arbeitsministerium gab in seiner Begründung zu dem Gesetzesvorhaben an, daß die berufliche Orientierung der Frauen sich verstärkt habe, ihre Lebensplanung sich diversifiziert hätte (womit es zugleich vom Schema des weiblichen Lebenszyklus abrückte) und schließlich durch die $\gg D e-$ kade der Frau« ein internationaler Handlungsdruck entstanden sei (vgl. Akamatsu 1985)

10 Ich verdanke diese Informationen Hinweisen von Prof. Park und Prof. Ujihara.

11 Die Überlegungen zur Bedeutung der Geschlechterpolarität in der alltäglichen Interaktion gründen sich neben einigen Betriebsstudien (vgl. u.a. Kawanishi 1981) auf eine Reihe von Interviews mit Führern der Betriebsgewerkschaften im Jahre 1985. Ein etwas bizarres Beispiel für »männlichen Arbeiterstolz« gab eine Gruppe von Elektroarbeitern auf der 1. Mai Demonstration 1979, die, parallel zum Anlaufen des »Supermann«-Filmes, auf ihren Plakaten versprach: »Unbeugsam wie Supermann werden wir kämpfen«. Ein Gewerkschaftsführer erwähnte als Problem des gewerkschaftlichen Aufstiegs von Frauen, daß sie aus der informellen Kommunikation bei den Trinkabenden ausgeschlossen seien und so schlechterer Zugang zu Informationen hätten.

12 Ich verdanke diese Information einem Hinweis von Gesine Foljanty-Jost.

13 Ein Forscherteam um Prof. Park stellte bei der Untersuchung von japanischen Firmen in der Bundesrepublik das Erstaunen des japanischen Managements darüber fest, daß Frauen nicht bei Heirat oder bei Geburt des ersten Kindes gekündigt werden können.

\section{Literatur:}

Akamatsu, Ryoko(1971): Sengo katei to fujin rodo no henka. In: Sengo fujin mondaishi. 2. Auf1. 1978, Tokyo, S. 97-113

dies. (1985): Danjo koyo kikai kintoho oyobi kaisei rodo kijunho, Tokyo

Beck-Gernsheim, Elisabeth (1986): Von der Liebe zur Beziehung? Veränderungen im Verhältnis von Mann und Frau in der individualisierten Gesellschaft. In: Johannes Berger (Hg.), Die Moderne Kontinuitäten und Zäsuren, Soziale Welt, Sonderband 4, S. 235-263 
Bergmann, Joachim (1983): Die Fragmentierung der Lohnarbeiterklasse in Japan. In: Leviathan l, S. $99-117$

Burawoy, Michael (1985): The Politics of Production, London

Cook, Alice; Hayashi Hiroko (1980). Working Women in Japan. Discrimination, Resistance and Reform, Ithaca

Doi, Takeo (1982): Amae. Freiheit in Geborgenheit, Frankfurt/M.

Erler, Gisela (1985): Frauenzimmer. Für eine Politik des Unterschieds, Berlin

Ernst, Angelika (1980): Japans unvollständige Vollbeschäftigung. Beschäftigungsprobleme und Beschäftigungspolitik. Mitteilungen des Instituts für Asienkunde 115, Hamburg

Herold, Renate (1980): Die Blume am Arbeitsplatz. Japans Frauen im Beruf, Tübingen

Hirano, Takako (1984): Gendai no joseikan. In: (Hg.) Joseigaku kenkyukai: Josei no imeeiji. Koza joseigaku 1., Tokyo, S. 1-24

Hobsbawn, Eric; Ranger, Terence (1983): The Invention of Tradition, Cambridge

Illich, Ivan (1984): Genus, Reinbek b. Hmg.

Ishida, Takeshi (1987): The Introduction of Western Political Concepts into Japan: Non-Western Societies' Response to the Impact of the West. In: Peripherie 27

Kawanishi, Hirosuke (1981): Kigyobetsu kumiai no jittai, Tok yo

Kemmochi, Kazushi (1983: Maikon kakumei to rodo no mirai, Tokyo

Kokusai joshi gakkai Hg. (1980): Gendai Nihon no shufu, Tokyo

Kumazawa, Makoto (1981): Nihon no rodoshazo, Tokyo

Lenz, Ilse (1981): Familienhaushalte, Sozialkosten und wirtschaftliches Wachstum. In: Gesine Foljanty-Jost (Hg.) u.a., Japans Sozial- und Wirtschaftsordnung im internationalen Kontext, Frankfurt/M., S. 177-217

dies., (1984): Kapitalistische Entwicklung, Subsistenzproduktion und Frauenarbeit. Der Fall Japan, Frankfurt/M.

dies., (1984a): Changes in Women's Role and the Sexual Division of Labour during the Japanese Modernization. In: Taka Bluhme-Kojima u.a. (Hg.), Structural Changes in Industrial Society in Japan and West Germany, Symposium Bielefeld

dies., (1987a): The Gender Factor in Industrial Employment and the Impact of Microelectronic Technology. Preliminary Remarks on the Case of Japan. In: Joachim Bergmann (Hg.), Shigeyoshi Tokunaga: Economic and Social Aspects of Industrial Relations. A Comparison of the German and the Japanese Systems. S. 129-153

dies., (1987b): Mikroelektronische Rationalisierung und Dynamisierung der Arbeitsmärkte: Herausforderungen für die japanischen Gewerkschaften. In: Pornschlegel (Hg.), Der Arbeitsmarkt und Kollektivverhandlungen in Gewerkschaftstheorien, Berlin

Nihon seisansei honbu (1985): Joshi shiniidai to koyo no shishin, Tokyo

Oba, Ayako; Ujihara Shojiro (1969): Fujin rodo. Gendai fujin mondai koza 2, 6. Aufl, Tokyo

Rodosho: Rodo hakusho

Rodosho fujinkyoku (1985): Showa 60 nenpan Fujin rodo no jitsujo, Tokyo

Said, Edward (1980): Orientalism. London

Shirai, Taishiro (1982): Die japanische Betriebsgewerkschaft. Berliner Beiträge zur sozial- und wirtschaftswissenschaftlichen Japanforschung 13, Bochum

Tanaka, Sumiko; Hitaka, Rokuro (1969): Fujin seisaku, fujin undo. Gendai fujin mondai koza 1, 4. Aufl. 1976, Tokyo

Ueno, Chizuko (1982): Shufu ronso o yomu, 2 Bde., Tokyo 